\title{
Rechtsgeschichte
}

http://www.rg-rechtsgeschichte.de/rg1

Zitiervorschlag: Rechtsgeschichte Rg 1 (2002)

$\operatorname{Rg} 2002$

$269-271$

http://dx.doi.org/10.12946/rg01/269-271

\section{Claudia Kraft}

\section{Männererbe}




\section{Männererbe*}

Karl Kaser formuliert in der vorliegenden Studie die These, dass das gleichberechtigte Männererbe in weiten Teilen des östlichen Europas die Struktur der dortigen agrarischen Gesellschaften nachhaltig geprägt hat. Er beginnt seine Darstellung um das Jahr I 500 und verfolgt die gesellschaftlichen Entwicklungen bis hinein in das zwanzigste Jahrhundert. Dabei sieht er in den spezifischen erbrechtlichen Regelungen bei der Bauern- und Hirtenbevölkerung dieser Großregion Europas ein Strukturelement, das die Haushaltskonstellationen, eine patriarchalisch bestimmte Familienideologie, familiale Arbeitsorganisation und Verwandtschaftsbeziehungen sowie die Ordnung der jeweiligen Lebensphasen nachdrücklich mitbestimmte und dessen Folgen zum Teil bis in die Gegenwart nachwirken. Der Autor schlägt einen weiten Bogen, indem er das gesamte östliche Europa in den Blick nimmt und dabei auch auf vergleichende Blicke nach West- und Südeuropa nicht verzichtet. Damit entsteht ein grundlegendes Überblickswerk, das die Analysekategorie »Erbgewohnheiten « für eine vergleichende europäische Geschichte operationalisiert. Besonders hervorzuheben ist, dass sich der Autor der Gefahr einer Verfestigung der konzeptionellen Dichotomie zwischen dem »Osten « und dem »Westen « bewusst ist, wenn er ein so komplexes und eine Vielzahl von Lebensbereichen prä- gendes Strukturelement wie den Erbfall zu einer Analysekategorie bei der Untersuchung unterschiedlicher Entwicklungswege in Europa macht. Er entgeht dieser Gefahr, indem er durch die differenzierte Darstellung der Erb- und Gesellschaftsstrukturen im östlichen Europa den Begriff des Ostens als Konstrukt einer westlich zentrierten Perspektive entlarvt, die ihren Untersuchungsgegenstand in unzulässiger Weise zu homogenisieren pflegt.

Bereits im ersten Kapitel drängt sich jedoch die Frage auf, ob hier nicht doch erneut - wenn auch auf reflektierter Grundlage - eine OstWest-Dichotomie perpetuiert wird. Die Erbgewohnheiten in Europa betrachtend kommt Kaser zu einer Aufteilung des Kontinents in eine West- bzw. Osthälfte, die relativ identisch mit der Ausdehnung der Ostkolonisation bzw. der Spaltung in West- und Ostkirche ist. Die so entstandene Grenzziehung erscheint gerade auf den ostmitteleuropäischen Raum bezogen zu grob, wird dieser doch damit (bis auf wenige Ausnahmen) zu einem Teil Westeuropas, was die derzeitigen Kandidaten für die EU-Osterweiterung sicher freuen dürfte, jedoch zu wenig tiefenscharf ist, wenn es um die historische Analyse der gesellschaftlichen und ökonomischen Entwicklung dieser Region geht.

Das zweite Kapitel wendet sich einer genaueren Analyse des gleichberechtigten Männer-

\footnotetext{
Karl Kaser, Macht und Erbe. Männerherrschaft, Besitz und Familie im östlichen Europa ( I 500-I900), Wien, Köln, Weimar: Böhlau 2000, 343 S., ISBN 3-205-98990-2
} 
erbes im östlichen Europa zu. Bei der Frage nach den Entstehungsgründen für die dominierenden Erbgewohnheiten kommt Kaser zu dem Ergebnis, dass es weniger staatliche administrative Maßnahmen oder etwa ökonomisch begründete Eingriffe der Feudalherren waren, die zur Verfestigung dieses Erbmusters beitrugen. Kulturelle Traditionen, die sich etwa in den Geschlechterverhältnissen widerspiegeln, erachtet der Autor als prägender. In diesem Zusammenhang zeigt sich eine gewisse Eindimensionalität der Untersuchung, die auch anderen Stellen zutage tritt. Mit der analytisch unscharfen Kategorie »Kultur « werden anscheinend nicht mehr zu hinterfragende Ergebnisse produziert. Dabei ist es gerade bei der Untersuchung von Geschlechterverhältnissen evident, dass auch Diskurse über dieselben ihrerseits die gesellschaftliche Wirklichkeit strukturieren. Somit wäre es interessant, bei der Untersuchung der Geschlechterverhältnisse nicht bei der reinen Deskription zu verharren, sondern auch die Wirkungsmacht von Geschlechtersymboliken in ihrem Einfluss auf realgeschichtliche Befunde zu untersuchen.

Die unterschiedlichen Folgen, die die Erbgewohnheiten für das gesellschaftliche Leben besaßen, arbeitet Kaser in dem Kapitel »Erbmuster und historische Ethnographie der Differenz« heraus. Wenn infolge des gleichberechtigten Männererbes der Besitz aufgeteilt wurde, die erbenden Söhne mit ihren Familien aufgrund von Landknappheit aber nicht den Familiensitz verließen, entstanden komplexe Haushaltsstrukturen, die sich in ihrer Arbeitsorganisation deutlich von den Kern- und Stammfamilien im südlichen und westlichen Europa unterschieden. Dass die Erklärungskraft des Parameters »Erbgewohnheiten « gerade für die beiden letzten Jahrhunderte nicht überschätzt werden sollte, zeigen Kasers Ausführungen zu aktuelleren gesellschaftlichen
Entwicklungen. So ist es zweifelhaft, ob es tatsächlich die stärkere Bindung an den Boden ist, die noch heute dafür sorgt, dass sich osteuropäische Erwerbsemigranten seltener zu einer dauerhaften Ausreise aus ihrem Heimatland entschließen. Ebenso wäre zu fragen, ob nicht das marode Gesundheits- und Fürsorgesystem der postkommunistischen Staaten und nicht so sehr die fortdauernde Altersautorität der Grund dafür ist, dass dort die Betreuung alter und kranker Personen noch immer vorwiegend in den Familien erfolgt.

Im vierten Kapitel wird der Persistenz der jeweiligen gesellschaftlichen Ordnungen nachgegangen. Kontinuität herrschte vor allem in spezifischen Ökosystemen wie etwa in durch Brandrodung bewirtschafteten Waldgebieten oder bei den Hirtengesellschaften des Balkans. Ursachen für rasche Veränderung sieht Kaser vor allem in den Einflüssen von Migration, Urbanisierung, in der Ersetzung gewohnheitsrechtlicher Grundlagen durch administrativ genormte Regeln sowie nicht zuletzt durch makroökonomische Eingriffe wie etwa die sozialistischen Kollektivierungen des 20. Jahrhunderts. Hier stellt sich die Frage, ob nicht bereits auch für frühere Perioden weitere gesellschaftliche Entwicklungsfaktoren hätten in die Untersuchung miteinbezogen werden sollen. So führt vielleicht die ausschließliche Konzentration auf die nichtstädtische Bevölkerung zu verzerrten Ergebnissen, bedenkt man, dass es zum Beispiel durchaus Transferleistungen zwischen Stadt und Land auch bereits im vormodernen Europa gegeben hat.

Die angeführten Kritikpunkte sollen den Wert der sehr differenziert geschriebenen Studie nicht schmälern. Kaser selbst ist sich durchaus der Gefahr seiner eindimensionalen Interpretation bewusst. In seinen zusammenfassenden Schlussbemerkungen warnt er erneut davor, aus 
den spezifischen Erbgewohnheiten des östlichen Europas ein allumfassendes Erklärungsmodell für dessen angebliche Rückständigkeit abzuleiten. Er stellt es weiteren Forschungen anheim, inwieweit etwa das »mobilitätshemmende gleichberechtigte Männererbrecht die spät einsetzende Urbanisierung und Industrialisierung mit verursachte oder ob spät einsetzende Urbanisierung und Industrialisierung die Erbgewohnheiten lange Zeit nicht in Frage stellten« (303). Nicht zuletzt im Hinblick auf aktuelle Debatten über die politische Neuordnung Europas nach dem Ende des Kalten Krieges wäre es interessant zu fragen, als wie resistent sich die ahnenzentrier- ten, patrilinearen Gesellschaftsmodelle gerade in den umstürzenden sozioökonomischen und politischen Veränderungen des 20. Jahrhunderts erwiesen. Die thesenartigen Erklärungsansätze Kasers etwa für gegenwärtige Entwicklungen im östlichen Europa, die er auf das Weiterwirken bestimmter traditioneller Erbgewohnheiten zurückführt, wären es wert, mit umfassenden empirischen Untersuchungen, die die tatsächliche Quantität des althergebrachten Traditionsbestandes in den postkommunistischen Gesellschaften herausarbeiten, überprüft zu werden.

Claudia Kraft 